\title{
Teaching Robot and Its Trajectory Planning Generation
}

\author{
Xiulan Wen*, Bingfeng Bai, Junyu Cui and Guifang Qiao \\ Automation Department, Nanjing Institute of Technology, Nanjing 211167, China \\ ${ }^{*}$ Corresponding author
}

\begin{abstract}
This paper focuses on designing a kind of six degree of freedom teaching robot (STROB) and developing its trajectory planning method. STROB structure is introduced and kinematics parameters model is established. In order to make STROB generate smooth and continuous trajectory, non-uniform Bsplines interpolation methods is analyzed and used to generate the robot's curved trajectory path. The simulation and practical application verify the proposed method effectiveness. The designed STROB can not only generate smooth and continuous trajectory path fast and smoothly, but also has the features that the robot body structure and control system are open and the inner structure of each joint is visible and debugged separately. It is suitable for popularization and application in teaching.
\end{abstract}

Keywords-teaching robot; non-uniform B-splines interpolation; trajectory planning

\section{INTRODUCTION}

The industrial robots have been widely used in the areas of spraying, palletizing, handling, packaging, welding, assembly, etc. Therefore, there is increasing demand for robot technology talents in recent years. The teaching robots become more and more important in teaching when they are treat as the carrier of the high technology [1]. It is meaningful to develop open structure design and control system of the teaching robot. It has great significance to popularize the applications of the industrial robotics, train technical talents and improve the level of the industrial automation. Trajectory planning for teaching robots is a very important issue in those motive activities which have been automated [2]. It can reduce the motor jitter to ensure the smooth operation of the robot. A good trajectory planning method should ensure that the robot operation is smooth, continuous and efficient. Therefore, trajectory planning for industrial robots is a very important topic and has attracted many researchers and some methods have been proposed. The early algorithm that solved the trajectory planning problem tried to minimize the time needed for performing the task [3-4]. Mattmuller determined smooth and near time-optimal path-constrained trajectories [5], in which not only velocity and acceleration but also jerk velocity were considered. In order to increase industrial applicability, Van Dijk [6] proposed a method to find the minimum time trajectory that accounted for robot kinematics together with actuator velocity, acceleration and jerk limits instead of accounting for the generally more complex dynamic equations of a manipulator with actuator torque and torque-rate limits. Lavalle [7] made an extensive work about planning algorithms in robotics, which included motion planning and trajectory planning among many other subjects. Rubio [8] proposed a new approach to solve the trajectory planning problem. And the idea was using a weighted objective function to optimize the operating parameters of the robot. Menasri proposed to use a genetic algorithm to solve robot trajectory planning and the result is verified by simulation [9]. Although scholars have proposed some methods in this area, the effectiveness of most methods were verified by simulation and few were applied to the robot's curved trajectory path.

In this paper, the application of teaching robot and the trajectory planning methods are introduced. STROB teaching robot is designed and the modified $\mathrm{D}-\mathrm{H}$ model (MDH) is established. Then, non-uniform B-splines interpolation method is analyzed and used to generate the robot's curved trajectory path. Finally, the experiments are made to prove the proposed method effectiveness and the results are included.

\section{STROB TEACHING ROBOT}

\section{A. STROB Structure}

Self-developed STROB is a six degree of freedom series robot shown in Fig. 1 and it consists of robot body, console, motors, encoder cable, power, industrial PC, PCI1040 motion control card, signal adapter board, software system, etc. Because most teaching robot body is close as a whole and students do not learn its inner structure, which will influence the latter part learning. In order to solve this problem, the control system and mechanical structure of STROB are open; each joint is designed separately and its inner structure is visible and debugged separately. Therefore, it is easy for students to learn the working principles of the motor, reducer and so on. Open STROB joints are shown in Fig. 2.

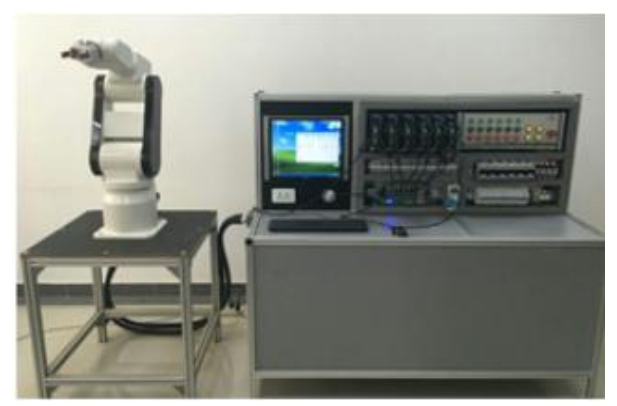




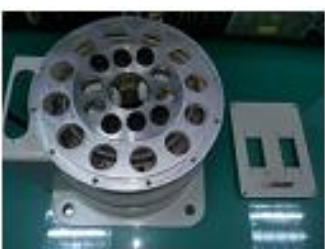

(a) ROBOT BASE

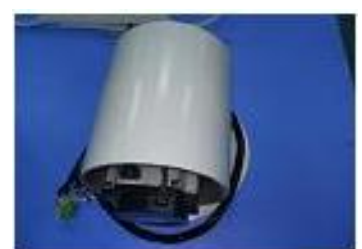

(b) SECOND JOINT
FIGURE.I. STROB TEACHING ROBOT

\section{B. Kinematics Parameter Modelling}

STROB is a joint robot and six joints are all rotary joints. The D-H parameters method proposed by Denavit and Hartenberg [10] is commonly used to establish the kinematic model. The classic D-H model is not only incomplete, but also the parameters are not continuous. If the actual adjacent joint axes are not parallel and they are modeled based on parallel joint axes in theory, the relative position error modeling between the two axes will not satisfy the "small error modeling" condition. It will result in parameters jump which directly influences the numerical computation stability in the process of subsequent parameter identification. In order to solve this problem, an additional rotation parameter $\beta$ for the parallel axes is introduced in MDH model [11]. The modified transformation matrix can be expressed as follows:

$$
\begin{aligned}
{ }_{i}^{i-1} T & =\operatorname{Rot}\left(Z_{i}, \theta_{i}\right) * \operatorname{Trans}\left(Z_{i}, d_{i}\right) * \operatorname{Trans}\left(X_{i-1}, a_{i-1}\right) * \operatorname{Rot}\left(X_{i-1}, \alpha_{i-1}\right) * \operatorname{Rot}\left(Y_{i}, \beta_{i}\right) \\
& =\left[\begin{array}{cccc}
c \theta_{i} c \beta_{i} & -s \theta_{i} & c \theta_{i} s \beta_{i} & a_{i-1} \\
s \theta_{i} c \alpha_{i-1} c \beta_{i}+s \alpha_{i-1} s \beta_{i} & c \theta_{i} c \alpha_{i-1} & s \theta_{i} c \alpha_{i-1} s \beta_{i}-s \alpha_{i-1} c \beta_{i} & -s \alpha_{i-1} d_{i} \\
s \theta_{i} s \alpha_{i-1} c \beta_{i}-c \alpha_{i-1} s \beta_{i} & c \theta_{i} s \alpha_{i-1} & s \theta_{i} s \alpha_{i-1} s \beta_{i}+c \alpha_{i-1} c \beta_{i} & c \alpha_{i-1} d_{i} \\
0 & 0 & 0 & 1
\end{array}\right]
\end{aligned}
$$

where $X_{i-1}$ is the $X$ axis of joint $i-1, Y_{i}$ is the $Y$ axis of joint $i, Z_{i}$ is the $Z$ axis of joint $i, \theta_{i}$ is the joint angle, $a_{i-1}$ is the length of connecting rod, $\alpha_{i-1}$ is the twist angle of connecting rod, $d_{i}$ is the offset of connecting rod, $s$ stands for $\sin , c$ stands for cos.

\section{Trajectory PlanNing Modeling Based On Non- UNIFORM B-SPLINES}

Non-uniform B-splines has the characteristics which retains the advantages of the Bezier curve and can solve the problem that the local of the Bezier curve is not easy to modify and the more control points lead to higher the curve order [12], therefore it is used to generate the trajectory planning in this paper.

The formula for non-uniform B-splines is as follows:

$$
p(u)=\sum_{i=0}^{n} d_{i} N_{i, k}(u)
$$

where $d_{i}(i=0,1,2 \ldots, n)$ is the controlling vertices, $N_{i, k}(u) \quad(i=0,1,2 \ldots, n)$ is the basis function of $k$ order specification $B$-splines and its value is obtained by the de-Cox recursive formula as follows:

$$
\left\{\begin{array}{l}
N_{i, 0}(u)= \begin{cases}1, & u_{i} \leq u<u_{i+1} \\
0, & \text { otherel se }\end{cases} \\
N_{i, k}(u)=\frac{u-u_{i}}{u_{i+k}-u_{i}} N_{i, k-1}(u)+\frac{u_{i+k+1}-u}{u_{i+k+1}-u_{i+1}} N_{i+1, k-1}(u), k \geq 1 \\
\text { Assuming } \quad \frac{0}{0}=0
\end{array}\right.
$$

where $k$ expresses the order of non-uniform $B$-splines, $i$ indicates the serial number of non-uniform $B$-splines, $u_{i}$ indicates the node. The node vector $U$ of non-uniform $B$ splines basis $N_{i, k}(u)$ is expressed as follows:

$$
\begin{aligned}
& U=[\underbrace{u_{0}, u_{1}, \cdots, u_{k}}_{k+1}, u_{k+1}, u_{k+2} \cdots u_{n}, \underbrace{u_{n+1}, \cdots, u_{n+k+1}}_{k+1}] \\
& =[\underbrace{a, a, \cdots, a}_{k+1}, u_{k+1}, u_{k+2} \cdots u_{n}, \underbrace{b, b, \cdots, b}_{k+1}]
\end{aligned}
$$

Usually, $a$ is set to $0, b$ is set to $1, u_{k+1}, u_{k+2} \cdots u_{n}$ is called as the inner nodes and can be computed by Riesenfeld method[13]. When $k$ is even number, the node vector $U$ is:

$$
U=\left[\begin{array}{l}
\underbrace{0,0, \cdots, 0}_{k+1}, \frac{\sum_{j=1}^{k / 2} l_{j}+\frac{l_{k / 2+1}}{2}}{L}, \frac{\sum_{j=1}^{k / 2+1} l_{j}+\frac{l_{k / 2+2}}{2}}{L}, \cdots, \\
\frac{\sum_{j=1}^{n-k / 2-1} l_{j}+\frac{l_{n-k / 2}}{2}}{L}, \underbrace{1,1, \cdots, 1}_{k+1}
\end{array}\right] .
$$

When $k$ is odd number, the node vector $U$ is:

$$
U=[\underbrace{0,0, \cdots, 0}_{k+1}, \frac{\sum_{j=1}^{(k+1) / 2} l_{j}}{L}, \frac{\sum_{j=1}^{(k+1) / 2+1} l_{j}}{L}, \cdots, \frac{\sum_{j=1}^{n-(k+1) / 2} l_{j}}{L}, \underbrace{1,1, \cdots, 1}_{k+1}] .(6)
$$

where $l_{i}=\left|d_{i}-d_{i-1}\right|(i=1,2, \ldots, n), L=\sum_{i=1}^{n} l_{i}$.

The teaching trajectory points can be obtained by controlling the robot movement and non-uniform B-splines control vertices can be computed according to these teaching points. In the process of computing the control vertexes, it is necessary to ensure that the derivatives of the curve are consistent with the corresponding data. Because there are only $k+1$ non-zeros $N_{j, k}(u)(j=i-k, i-k+1, \ldots, i)$ at most, the $k$ order non-uniform B-splines curve equation which is used to interpolate $n+1$ points can be written as: 


$$
\begin{aligned}
& p(u)=\sum_{j=i-k}^{i-l} d_{j} N_{j, k-l}(u)=\cdots=d_{i-k}^{k}, \\
& u \in\left[u_{i}, u_{i+1}\right] \subset\left[u_{k}, u_{n+1}\right] \\
& d_{j}^{l}=\left\{\begin{array}{lc}
d_{j}, & l=0 \\
\left(1-a_{j}^{l}\right) d_{j}^{l-1}+a_{j}^{l} d_{j+1}^{l-1}, j=i-k, i-k+1, \cdots i-l ; l=1,2, \cdots, k
\end{array}\right. \\
& a_{j}^{l}=\frac{u-u_{j+1}}{u_{j+k+1}-u_{j+l}}, \quad \text { Assumming } \quad \frac{0}{0}=0
\end{aligned}
$$

The $r$ order derivative vector of a point on B-splines can be deduced as follows:

$$
\begin{aligned}
& p^{r}(u)=\sum_{j=i-k+r}^{i} d_{j}^{r} N_{j, k-r}(u), \quad u_{i} \leq u \leq u_{i+1} \\
& d_{j}^{l}=\left\{\begin{array}{lc}
d_{j} & l=0 \\
(k+1-l) \frac{d_{j}^{l-1}-d_{j-1}^{l-1},}{u_{j+k+1-l}-u_{j}} & l=i-k, 2, \cdots, r
\end{array} .\right.
\end{aligned}
$$

where $u_{i+k} \in\left[u_{k}, u_{n+k}\right], i=0,1, \cdots n$.

Therefore, the $r$ order derivative vector of $k$ order Bsplines can be expressed $k-r$ order $\mathrm{B}$-splines and the controlling vertices can be calculate recursively. The position, velocity, acceleration and jerk of B-splines fitting trajectory at any time are calculated by (7) and (8). If the $k$ times B-splines is open curve, the $k-1$ tangent vector boundary equations are required and the starting and ending points are required data, we have:

$$
\begin{gathered}
\dot{p_{0}}=\dot{p}\left(u_{k}\right)=\sum_{j=1}^{k} d_{j}^{1} N_{j, k-1}\left(u_{k}\right)=d_{1}^{1} \\
\vdots \\
\dot{p_{n}}=\dot{p}\left(u_{n+k}\right)=\sum_{j=n}^{n+k-1} d_{j}^{1} N_{j, k-1}\left(u_{n+k}\right)=d_{n+k-1}^{1} \\
\ddot{p_{0}}=\ddot{p}\left(u_{k}\right)=\sum_{j=2}^{k} d_{j}^{2} N_{j, k-2}\left(u_{k}\right)=d_{2}^{2} \\
\vdots \\
\ddot{p_{n}}=\ddot{p}\left(u_{n+k}\right)=\sum_{j=n+1}^{n+k-1} d_{j}^{2} N_{j, k-2}\left(u_{n+k}\right)=d_{n+k-1}^{2}
\end{gathered}
$$

TABLE I. THE KEY TEACHING POINTS OF ROBOT TRAJECTORY

\begin{tabular}{cccc}
\hline Number & $\mathrm{X}(\mathrm{mm})$ & $\mathrm{Y}(\mathrm{mm})$ & $\mathrm{Z}(\mathrm{mm})$ \\
\hline 1 & -105.965 & -425.002 & 200 \\
2 & -97.813 & -423.673 & 200 \\
3 & -89.712 & -420.165 & 200 \\
4 & -81.672 & -417.990 & 200 \\
5 & -73.703 & -415.541 & 200 \\
6 & -65.815 & -412.816 & 200 \\
7 & -58.017 & -410.379 & 200 \\
8 & -43.113 & -410.421 & 200 \\
9 & -40.435 & -411.634 & 200 \\
10 & -43.257 & -413.920 & 200 \\
11 & -45.683 & -415.367 & 200 \\
12 & -44.061 & -420.015 & 200 \\
13 & -44.017 & -425.890 & 200 \\
14 & -38.082 & -423.232 & 200 \\
15 & -35.672 & -418.497 & 200 \\
16 & -23.846 & -412.207 & 200 \\
\hline
\end{tabular}

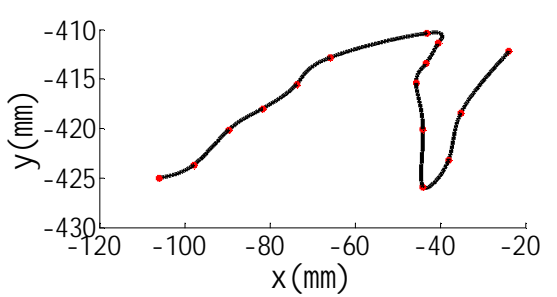

FIGURE II. ROBOT MOVEMENT TRAJECTORY
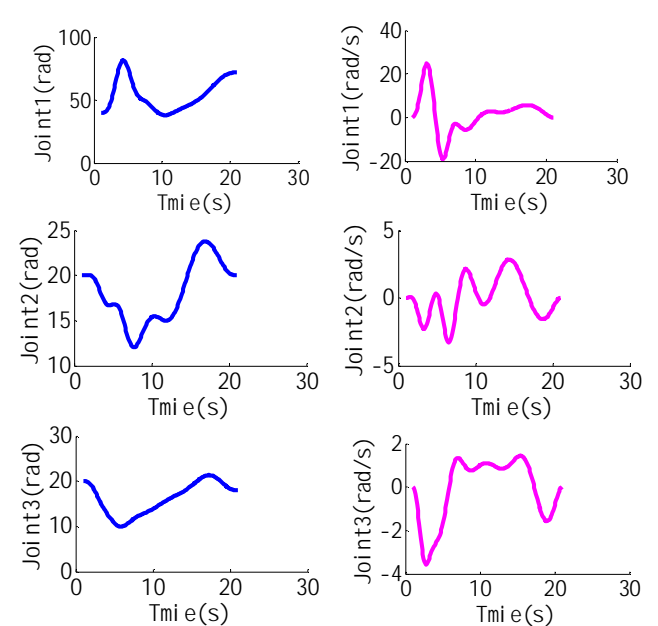

(a) angle

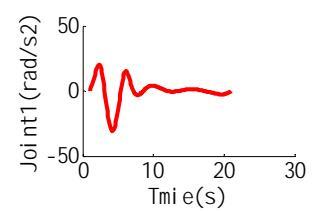

(b) angular velocity

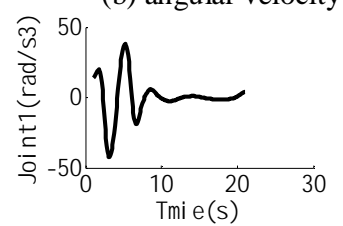



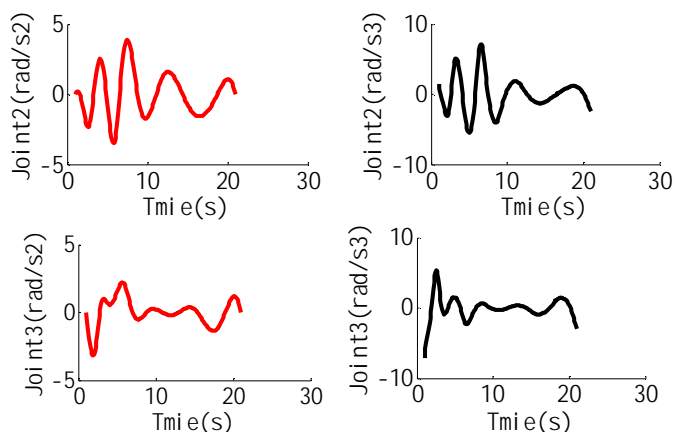

(c) angular acceleration

(d) jerk velocity

FIGURE III. THE TRAJECTORY PLANNING CURVES OF 1-3 JOINTS

\section{SIMULATION AND EXPERIMENT}

The fifth order B-splines is used to generate the trajectory curve for the robot. The angle of each joint is obtained by reading the value of the encoder. The key teaching points are computed by robot positive kinematic solution and are listed in Table 1 and the trajectory of these teaching points generated by using the fifth B-splines is shown in Fig.2.

The angle, angular velocity, angular acceleration and jerk velocity of each joint are obtained and Figure 3 draws a diagram of three joints. It can be seen the angular displacement, velocity, acceleration and jerk velocity of three joints are all smooth and continuous.

The trajectory planning practical application of the STROB by using the fifth B-splines is shown in Fig.4. During the process of experiments, Lenovo B470 computer is used. Its processor is the Inter core $\mathrm{i} 3$, the main frequency is $2.2 \mathrm{GHz}$ and memory is $2 \mathrm{G}$. It took $0.94 \mathrm{~s}$ to generate the movement trajectory of Fig.2 by the fifth B-splines on Matlab 8.1 platform. STROB has three kinds of speed modes, which are low, medium and high speed. When the medium speed is set, it took about $4.5 \mathrm{~s}$ for STROB to go through the path shown in Fig.4. It can be seen that the robot can go through the twists and turns trajectory smoothly, continuously and quickly. It can satisfy the actual need.

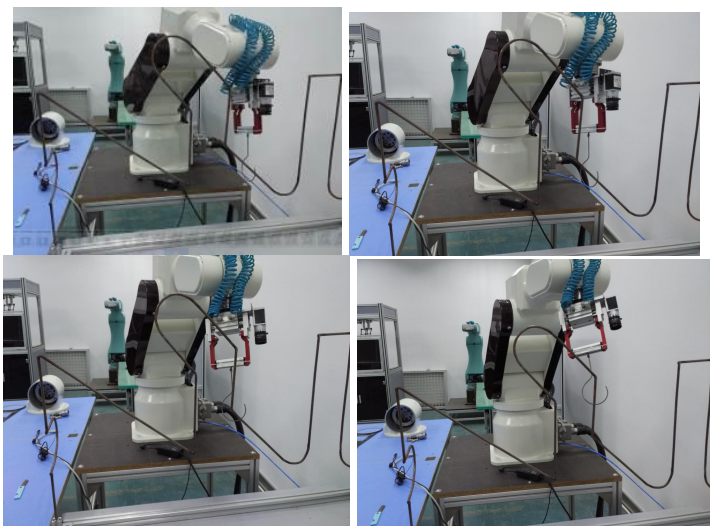

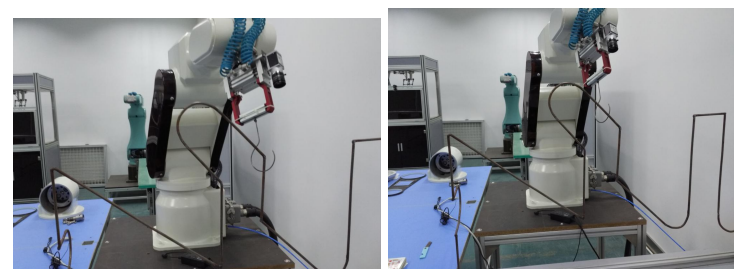

FIGURE.IV. THE ACTUAL TRAJECTORY OF STROB

\section{CONCLUSION}

In this paper, a kind of six degree of freedom teaching robot is designed and its trajectory planning modeling method is proposed. In order to make the student learn the inner structure, the STROB structure is designed open; each joint is visual and debugged separately. The fifth B-spliness interpolation method is analyzed and simulated on the MATLAB platform. The simulation results show that it can ensure that the velocity, acceleration and jerk velocity of robot trajectory planning curve are smooth and continuous. And it is used to generate STROB trajectory and it can go through the twists and turns trajectory smoothly, continuously and quickly. The results verify that the self-developed STROB can meet the teaching requirements. It is easily popularized and applied in teaching.

\section{ACKNOWLEDGMENT}

The authors are indebted to the referees for several suggestions that substantially improved the paper. The research was supported by Natural Science Research Project of the People' s Republic of China (No: 51675259), Natural Science Research Project of Jiangsu Province (16KJB460013).

\section{REFERENCES}

[1] Sato T, Fudaba Y, Tsusaka Y. "Teaching device for robot, robot apparatus, method of controlling teaching device for robot, and control program of teaching device for robot", US 9211640 B2[P]. 2015.

[2] K.Kaltsoukalas,S.Makris,G.Chryssolouris, "On generating the motion of industrial robot manipulators,"Robot.Comput.-Integr.Manuf. vol.32, no.1, pp.65-71, 2015.

[3] K.G. Shin, N.D. McKay, "Minimum-time control of robotic manipulators with geometric path constraints," IEEE Trans. Automat. Control (ISSN: 0018-9286), pp.531-541, 1985.

[4] Yaobin Chen, Alan A. Desrochers. "Minimum-time control laws for robotic manipulators,'International Journal of Control, pp.57-59, 1993.

[5] J. Mattmuller, D. Gisler, "Calculating a near time-optimal jerkconstrained trajectory along a specified smooth path,” Internat. J. Adv. Manufac. Technol. vol.45, no.2, pp.1007-1016, 2009.

[6] N.J.M. van Dijk, N. van de Wouw, H. Nijmeijer, W.C.M. Pancras, "Pathconstrained motion planning for robotics based on kinematic constraints," Proceedings of the ASME International Design Engineering Technical Conferences and Computers and Information in Engineering Conference, vol. 8, pp.1071-1080, 2008.

[7] S.M. Lavalle, "Planning Algorithms," Cambridge University press, UK, 2006.

[8] F.J. Rubio, C. Llopis-Albert, F.J. Valero, J.L. Suer, "Assembly line productivity assessment by comparing optimization-simulation algorithms of trajectory planning for industrial robots," Math. Probl. Eng. http://dx.doi.org/10.1155/2014/931048, 2014.

[9] Menasri R, Oulhadj H, Daachi B, et al.“A genetic algorithm designed for robot trajectory planning", IEEE International Conference on Systems, Man and Cybernetics, 2014(06), pp.228-233. 
[10] Denavit, Hartenberg R S. "A kinematic notation for lower-pair mechanisms based on matrices". Joernal of Applide Mechanics, 1955, pp. 215-221.

[11] ZHOU Gang, ZHANG Tie."Study on Robot Calibration Algorithm Based on MDH Model”. Computer \& Digital Engineering, 2010, pp.1719.

[12] Ying Yue,YanBo Yue. "Kinematics Simulation and Trajectory Planning of Six Degrees of Freedom”. Combination Machine Tool and Automatic Machining Technology, 2016(04), pp.89-92.

[13] F.H.Shi, Computer aided geometric design \& nonuniform rational Bspliness [M], Higher education press, 2001 (in Chinese) 